\title{
Biochemical Studies on Carbohydrates.
}

CXXI. On the Direct-Ehrlich Reaction-giving Structure in Glycidamins.

First Report.

By

\author{
Noboru Hiyama. \\ (檜山 登)
}

(From the Medico-chemical Institute, Tohoku University, Sendai.

Director: Prof. H. Masamune.)

(Received for publication, August 25, 1948)

- Blix ${ }^{11}$ boiled salivary mucin with water and isolated from the extract a crystalline acid ("Kohlenhydrat I") analyzing $42.62-44.70 \%$ C, $6.11-$ $6.61 \% \mathrm{H}$ and $3.68-4.41 \% \mathrm{~N}$. This substance gave Ehrlich reaction and with dilute $\mathrm{FeCl}_{3}$ a yellow coloration and reduced hot alkaline copper solution. Its neutral equivalent was 381-388 and two molecules of acetic acid were set free when it was heated with $p$-toluenesulfonic acid. On these facts the author assigned it $\mathrm{C}_{14} \mathrm{H}_{24} \mathrm{NO}$ as the most probable formula.

It is a very fascinating problem to clear up the direct-Ehrlich reactiongiving structure which is detected in most glycidamins (cf. Osaki and Turumi $\left.^{2}\right)$. For the solution of this problem Blix's substance makes an appropriate means. Despite attempts, the writer could obtain it only in an oily form but not as crystals. Since, however, the product showed the chemical-qualitative properties of the crystals Blix described, it was taken as a material for the study. Many trials to separate the structure in question failed, until, finally, treatment with hot strong alkali afforded a crystalline product that reacts with $p$-dimethylaminobenzaldehyde (the direct Osaki-Turumi process) like as the mother substance, giving a colour, though somewhat divergent in nuance and spectrum. The spectrum of the colour (purplish) from the product possessed absorption between 530$555 \mathrm{~m} \mu$ of $\lambda$ (max. absorp. $550 \mathrm{~m} \mu$ ), whereas, when the Blix's substance is tested, the corresponding colour (red) showes an absorption band between $540-580 \mathrm{~m} \mu$ (max. absorp. $565 \mathrm{~m} \mu$ ). It showed also a pine shaving test (A pine shaving moistened with conc. hydrochloric acid is dipped in a solution of the substance). A small yield did not allow a farther-going examination so that the salivary mucin itself was next resorted to, and a substance identical (qualitative and mixed melting point tests) with the 
above was obtained in a quite sufficient amount and demonstrated to be pyrrole- $\alpha$-carboxylic acid (identified as such and as methylester). That it may have originated in glucosamine or the amino-acid groupings of the mucin was excluded by experimenting with caseinogen and gelatine, which are non-glycidamin simple proteins, and with glucosamine hydrochloride. These proteins gave traces of a substance, which gave the positive pine shaving test for pyrrole and colour reaction with Ehrlich aldehyde, but in the latter reaction the tone and spectrum of the colour differed from those of the colour from pyrrole- $\boldsymbol{\alpha}$-carboxylic acid. Further divergencies of this substance will be described in the experimental part. It looks probable that this pyrrole compound from caseinogen and gelatine was also produced from the mucin but was missed due to its large solubility in chloroform (cf. below). From glucosamine hydrochloride crystals were secured which were in accord with pyrrole- $\alpha$-carboxylic acid in solubility, crystalline form, sublimability and the form of crystals thereby condensing, and the spectrum of the colour that developed by the Ehrlich reaction. But the yield of this probable pyrrole- $\alpha$-carboxylic acid was too small (even less than $1 \mathrm{mgm}$. from $20 \mathrm{gm}$. of glucosamine hydrochloride) to assume that, in the experiments on the mucin and the crude Blix's substance, most of pyrrole- $\alpha$-carboxylic acid came from simple glucosamine.

From these observations, following chemism is surmised as regards production of pyrrole- $\alpha$-carboxylic acid (II) from the Blix's substance.
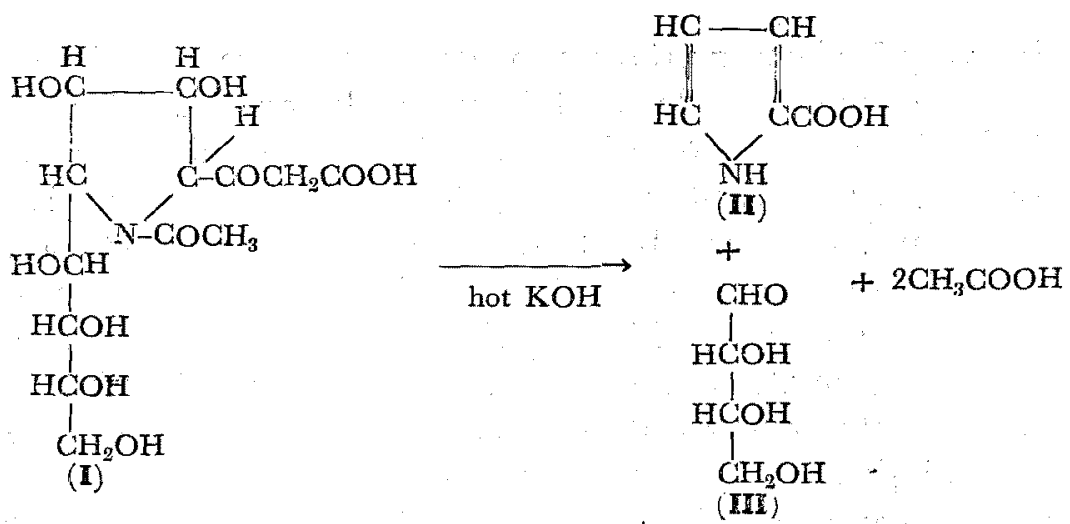

To the Blix's substance is here tentatively alloted structure $\mathbf{I}$, which satisfactorily explains its properties already quoted. The percentages of $\mathrm{C}, \mathrm{H}$ and $\mathrm{N}$ calculated from it $\left(\mathrm{C}_{13} \mathrm{H}_{21} \mathrm{O}_{10} \mathrm{~N}\right)$ amount to 44.4, 6.0 and 4.0 , nearly coinciding with the analyses of Blix. Although the equivalent weight (351) according to this formula does not reach the value observed (381-388), the author took too small amounts for accurate determination. As to its direct Ehrlich reaction, cleavage between 2-C and 3-C of the glucosamine chain in $\mathbf{I}$ and simultaneous dehydration will occur on 
heating with the reagent, giving a pyrrole derivative which condenses with the aldehyde. In the course of separating pyrrole- $a$-carboxylic acid the hot strong alkali might have split off the acetyl rests besides. Reduction of hot alkaline copper solution by the Blix's substance is ascribed to $d$ erythrose (III) formed from the latter.

\section{EXPERIMENTAL.}

Separation of Pyrrole- $\alpha$-carboxylic Acid.

The run started with salivary mucin will be described in full, because the procedure here was typical and the product was most thoroughly examined.

\section{Separation from salivary mucin.}

1) The procedure.

Sublingual mucin was prepared from the bull gland according to Hammarsten $^{3}$. It was boiled for 1 hour with 8 volumes of alcohol (95\%), washed with hot alcohol and dried in vacuum. $20 \mathrm{gm}$. of this material were taken up in $200 \mathrm{cc}$. of $10 \% \mathrm{KOH}$ and boiled gently in the stream of $\mathrm{N}_{2}$-gas for 10 hours under reflux. The dark brown digestate removed from some black sediments was diluted to $300 \mathrm{cc}$. and, under cooling, $10 \% \mathrm{H}_{2} \mathrm{SO}_{4}$ added in small portions to weakly alkaline ( $\mathrm{pH} 7.0-7.4)$. After evaporating the solution under diminished pressure, the residue was boiled with three $80 \mathrm{cc}$. portions of $95 \%$ alcohol (boiled each time for 30 minutes). These extracts were filtered and distilled together at a. low temperature. The remaining dark thick syrup was dissolved in $50 \mathrm{cc}$. of water $(\mathrm{pH}$ of the solution was weakly alkaline), exhausted with ether and then cautiously acidified to $\mathrm{pH} 2.0$ with $20 \% \mathrm{H}_{2} \mathrm{SO}_{4}$. Centrifuged. The centrifugate was washed with a little water and the supernatant liquid and washing united were shaken with five $50 \mathrm{cc}$. portions of ether. The combined extracts were shaken with about $200 \mathrm{cc}$. of the saturated $\mathrm{Ba}(\mathrm{OH})_{2}{ }^{4}$. Then the watery layer was separated, removed from the excess barium by means of $\mathrm{CO}_{2}$ and subjected to vacuum distillation to about $100 \mathrm{cc}$. After decolorization with activated charcoal, it was condensed furtheron to syrupy consistency, eliminating half way some precipitate that appeared. To the remainder was added $96 \%$ alcohol as far as precipitation happened and, after one day, precipitates were collected by centrifugation, washed with alcohol and ether and dried in vacuo. The yellowish powder that was given $(2.2 \mathrm{gm})$ proved to contain a considerable amount of barium acetate. Therefore it was dissolved in $50 \mathrm{cc}$. of water and the insoluble part having been discarded, most of barium 
was precipitated out with sulfuric acid $(0.5 \mathrm{n}$.$) . And the centrifuged$ supernatant liquid and washing of the centrifugate were united and exhausted with ether. The extract dried with anhydrous sodium sulfate was evaporated in a desiccator $(\mathrm{KOH})$ by means of a jet pump, whereby acetic acid was removed. Brown-stained crystals and resinous substance remained which were stood with chloroform overnight and washed with chloroform on a suction funnel. The crystals obtained undissolved were $25 \mathrm{mgm}$.

Purification. The crude crystals were dissolved in $5 \mathrm{cc}$. of water by the aid of the saturated $\mathrm{Ba}(\mathrm{OH})_{2}{ }^{5}$. The slightly alkaline solution was treated with charcoal and a just enough quantity of sulfuric acid ( 1 and 0.1 n. solutions) to precipitate all barium was added and the mixture was treated, immediately without separating off of the precipitates, with ether. The extract was evaporated to about $5 \mathrm{cc}$. and transferred to a small Erlenmeyer flask. When its volume decreased to $0.5-1.0 \mathrm{cc}$. on standing in air, crystals in tetragonal (rhombic) and hexagonal plates began to come out. The vessel was stoppered hereupon and full crystallization awaited. Followingly ice-cooled $50 \%$ alcohol equal in volume to the content was added and the crystals collected on a suction funnel, washed with the dilute alcohol similarly cooled, and dried in a desiccator $\left(\mathrm{CaCl}_{2}\right)$ under normal pressure. Yield, $20 \mathrm{mgm}$.

From two $20 \mathrm{gm}$. and two $30 \mathrm{gm}$. portions of the salivary mucin were produced in a like way $83 \mathrm{mgm}$. of pure crystals altogether.

2) Properties of the product above.

It was readily soluble in methanol, ethanol and ether, and soluble with less ease in water and with difficulty in chloroform.

A red colour developed on treatment with $p$-dimethylaminobenzaldehyde by the direct process of Osaki and Turumi. This colour showed a spectrum with an absorption band from 530 to $555 \mathrm{~m} \mu$ of $\lambda$ (max absorp. near $550 \mathrm{~m} \mu$ ). Pine shaving-a pine shaving was moistened with concentrated hydrochloric acid and immersed in the solution of the product ${ }^{6}$ ), whereby violetred colour appeared-and sodium nitroprusside tests for pyrrole were given. N (Lassaigne) was detected. It did not reduce hot Fehling solution.

By the usual method (an unsealed capillary used) of melting point test, it sintered at $185-190^{\circ} \mathrm{C}$ and, on further elevation (slow) of temperature, decomposed at $204-205^{\circ} \mathrm{C}$ under gas evolution. Simultaneously crystals in hexagonal plates and rods condensed at the upper part of the tubelet by sublimation. When the capillary $(2 \mathrm{~cm}$.) was sealed according to Ciamician and Silber, ${ }^{7}$ ) it melted at $191.5-192^{\circ} \mathrm{C}$ under decomposition, and its mixtures with synthetical pyrrole- $\alpha$-carboxylic acid (s. below.) and 
with the product from " Kohlenhydrat I" (oily product, s. below) showed no depression of melting point. On burning it left no ash.

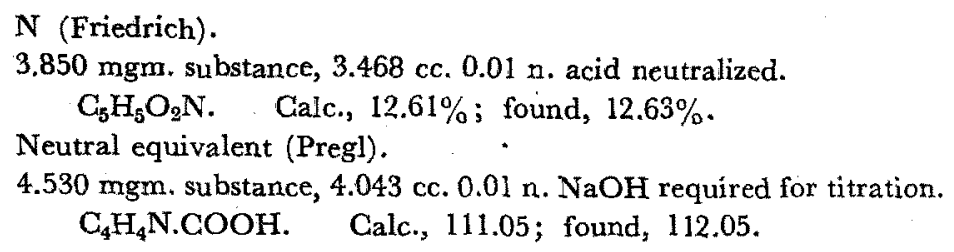

By Suzuki's modification ${ }^{81}$ of Friedrich, Rapoport and Sternberg method for acetyl determination, the substance gave a volatile acid amounting to $3.98 \%$ as acetyl $(4.120 \mathrm{mgm}$. substance, $0.378 \mathrm{cc} .0 .01 \mathrm{n}$. thiosulfate used for titration). This owed probably to carboxyl partially cleft by toluenesulfonic acid.

Methylester was derived according to Schwanert." . From $70 \mathrm{mgm}$. of the product above $125 \mathrm{mgm}$. of silber salt and $31 \mathrm{mgm}$. of the ester (long bevelled prisms; F.P., $73^{\circ} \mathrm{G}$ ) were given. The mixed melting point test with the corresponding ester of synthetical pyrrole- $\alpha$-carboxylic acid ( $F$. P. $73^{\circ} \mathrm{C}$, s. below) showed no depression.

N (Friedrich).

$5.247 \mathrm{mgm}$. substance, $4.148 \mathrm{cc} .0 .01 \mathrm{n}$. acid neutralized.

$\mathrm{C}_{6} \mathrm{H}_{7} \mathrm{O}_{2} \mathrm{~N}$. Calc., $11.2 \%$; found, $11.07 \%$.

\section{Separation from a "Kohlenhydrat I" (Blix) (oily product).}

To get the Blix's substance two $30 \mathrm{gm}$. portions of salivary mucin (submaxillary mucin) were subjected to his preparation procedure at first separately and half way combining the methanol extracts of their fraction, which had been dissolved in boiling water. The oily precipitates from methanol on addition of ether and petroleum ether did not change into crystals. Hence they were dried as such in a vacuum desiccator $\left(\mathrm{CaCl}_{2}\right)$ and $1.9 \mathrm{gm}$. of brownish glassy bulk were given. Blix obtained only $30-$ $60 \mathrm{mgm}$. of the crystalline substance from $40 \mathrm{gm}$. of the mucin. Therefore the above large yield suggested extreme impureness of the product which, however, was revealed similar in qualitative respects as follows:-

Biuret, ninhydrin, Millon and Molisch tests resulted all in negative. The direct Ehrlich reaction by the Osaki-Turumi technique showed red coloration (max. absorp. $565 \mathrm{~m} \mu$ ). By dilute $\mathrm{FeCl}_{3}$ appeared yellow colour. Hexosamine reaction was demonstrated, though feeble, by the quantitative estimation method of Hamasato and Akakura. The substance reduced hot Fehling solution. In addition, vanilline-hydrochloric acid test of Kobayasi ${ }^{10)}$ and glacial acetic acid-hydrochloric acid test of the writer ${ }^{11)}$ gave cherry-red and brownish red colour respectively.

$1.8 \mathrm{gm}$. of the above substance were treated like as the mucin, but 
the digestate with $10 \% \mathrm{KOH}$ was adapted to ether extraction without preliminary fractionation by means of alcohol. $5 \mathrm{mgm}$. of crystals in hexagonal plates were given. This substance exhibited the direct Ehrlich reaction (Osaki-Turumi process employed). Red coloration with purplish shade took place and absorption extended from about 530 to $555 \mathrm{~m} \mu$ of $\lambda$. In a sealed tubelet it melted at $190^{\circ} \mathrm{G}$ (decomp.) and the mixed melting point tests with the immediate product from the mucin as well as with synthetical pyrrol- $\alpha$-carboxylic acid exhibited no depression. In a capillary unsealed, it melted at $204^{\circ} \mathrm{C}$ (decomp.) and sublimed in part, condensing in crystalline forms with the same appearance as in the case of the other products just mentioned.

$\mathrm{N}$ (Friedrich).

$3.097 \mathrm{mgm}$. substance, $2.744 \mathrm{cc} .0 .01 \mathrm{n}$. acid neutralized.

$\mathrm{C}_{5} \mathrm{H}_{5} \mathrm{O}_{2} \mathrm{~N}$. Calc., $12.61 \%$; found, $12.40 \%$.

Synthesis of Pyrrole- $\alpha$-carboxylic Acid.

It was prepared with pyrrole and ammonium carbonate according to Ciamician and Silber, but the crude crystals (F.P. in an unseảled tube, $155-158^{\circ} \mathrm{C}$ ) were washed with cold $95 \%$ alcohol, whereby the melting point was raised at once to $193-195^{\circ} \mathrm{C}$, and further subjected to the procedure adopted to purify the product from the mucin (s. the paragraph "Purification"). The methylester was made by strictly following the method of Schwanert. The preparations both proved authentic.

Trials to See if Pyrrole- $\alpha$-carboxylic Acid is Obtainable from Gelatine, Caseinogen and Glucosamine Hydrochloride.

An experiment on gelatine.

$40 \mathrm{gm}$. of gelatina alba pulvis E. Merck were taken. The material did not react with $p$-dimethylaminobenzaldehyde at all by the direct as well as indirect-process of Osaki and Turumi. Subjected to the cleavage with $\mathrm{KOH}$ and following fractionation described in the case of the mucin, it gave $215 \mathrm{mgm}$. of a mixture of barium salts, which showed the positive direct-Ehrlich reaction, but after removal of barium, the ether extract left on evaporation only brown resinous substance with few crystal-like granules. On repetition of dissolving in water (the insoluble part separated off), extracting with ether and evaporation, the remainder (about $45 \mathrm{mgm}$.) looked like before (pyrrole- $\alpha$-carboxylic acid would crystallize beautifully at this stage). No suitable solvent was found to separate the granules (these were soluble in chloroform) so that the remainder was tested as a whole:- 1) It gave intensive direct Ehrlich reaction (Osaki-Turùmi process). Nevertheless, the developing colour (purple) had different 
nuance from the one (purplish red) that appears when pyrrole- $\alpha$-carboxylic acid reacts, and its absorption band lay between 540 and $600 \mathrm{~m} \mu$ of wave length instead of $530-555 \mathrm{~m} \mu$ in the latter. 2) Pine shaving reaction by the technique described-positive (red coloration). 3) Silver nitrate precipitated a solution of its barium salt as in the case of pyrrol- $\alpha$-carboxylic acid. But the deposit (This gave the characteristic colour by $p$-dimethylaminobenzaldehyde) dissolved rather easily in plain water in contradistinction to the corresponding salt of pyrrole- $\alpha$-carboxylic acid. 4) It was precipitated from water with lead acetate (the precipitates were similar to above regarding the product by Ehrlich reaction), while pyrrole- $a$ carboxylic acid remains in solution. These findings indicated that, from gelatine, a pyrrole compound but not pyrrol- $\alpha$-carboxylic acid is produced, although its exact nature could not be established due to too small a crop.

\section{An experiment on caseinogen.}

Hammarsten's caseinogen E. Merck was used. A red colour developed by the $p$-dimethylaminobenzaldehyde reagent (the direct as well as indirect process of Osaki and Turumi) but this reaction was also given by the mere solvent of the reagent. Faint Molisch reaction took place.

Similar operations were applied to $40 \mathrm{gm}$. of this protein as to the mucin and gelatine. A mixture of barium salts, that was obtained, gave weak Ehrlich reaction and weighed $630 \mathrm{mgm}$. It was almost free from phosphates (ammonium molybdate test). The evaporated residue of its ether extract consisted only of homogeneous glutinous substance. Ether extraction after fractioning with water and evaporation was repeated as in the foregoing experiment, but it caused no crystallization. The product $(65 \mathrm{mgm}$.) perfectly agreed with the one from gelatine in Ehrlich reaction particularly as to the spectrum of the colour developing hereby, in the pine shaving reaction and in solubility of the silber salt.

\section{An experiment on glucosamine hydrochloride.}

$20 \mathrm{gm}$. of glucosamine hydrochloride were treated with $200 \mathrm{cc}$. of hot $10 \% \mathrm{KOH}$ with following processes as before. The separated grayish extraordinarily hygroscopic barium-salt mixture amounted to $1.4 \mathrm{gm}$. It reacted with the $p$-dimethylaminobenzaldehyde reagent (Osaki and Turumi) giving red colour" with purplish hue. The spectrum had an absorption band from about 520 to about $570 \mathrm{~m} \mu$. Pine shaving reaction in the special way (s. above) and sodium nitroprusside test for pyrrole resulted also in positive. The ether extract of the salt mixture (the water-insoluble part separated off) after removal of barium gave only a brown resinous residue that remained homogeneous on standing, but in the evaporated rest 
after extracting it again from water ${ }^{12}$ appeared a few hexagonal crystals resembling the crystals of pyrrole- $\alpha$-carboxylic acid. By washing with chloroform the crystals ${ }^{13}$ ) were separated. The total amount did not reach beyond $1 \mathrm{mgm}$. Heating of it to $180^{\circ}$ gave rise to sublimation and condensation into tetragonal and hexagonal crystals as when pyrrole- $\alpha$-carboxylic acid is treated, and these crystals gave the direct Ehrlich reaction with coloration peculiar to this acid (the spectrum showed absorption from 530-555 $\mathrm{m} \mu$ ) and were not precipitated from water by lead acetate but were by silber nitrate after neutralizing with baryta. Lack of substance did not allow further investigation.

Through the Grant Committee of National Research Council a grant was given from the Education Department in aid to us, which is gratefully acknowledged.-Masamune.

\section{Bibliography and Notes.}

1) Blix, Zeits. f. physiol. Chem., 1936, $240,43$.

2) Osaki and Turumi, Tohoku J. Exp. Med., 1947, 49, 11.

3) Hammarsten, Zeits. f. physiol. Chem., 1888, 12, 163.

4) When the water layer did not remain alkaline after shaking with the ether extracts, the baryta solution was supplemented.

5) A large volume of water is required to dissolve them without the use of alkali.

6) By this technique the test was negative not only in salivary mucin but also in gelatine and caseinogen.

7) Ciamician and Silber, Ber. dtsch. chem. Ges, $1884,17,1150$.

8) Suzuki, M., J. Bioch., 1938, 27, 367.

9) Schwánert, Ann. d. Chem., 1860, 116, 270.

10) Kobayasi, J. Bioch., 1941, 34, 145 (footnote).

11) Hiyama, Tohoku J. Exp. Med., in press.

12) A part of the residue was found insoluble. This was rejected and the solution treated with charcoal.

13) They were still coloured brown. 